\title{
What is unknown about the statistics of extrasolar planetary systems?
}

\author{
Hugh R.A. Jones \\ Astrophysics Research Institute, Liverpool John Moores University, Twelve Quays House, \\ Egerton Wharf, Birkenhead CH41 ILD, UK
}

\begin{abstract}
While the study of extrasolar planetary systems has made astonishing progress during the last decade we are very much at the beginning of this new topic. Our study so far has only been sensitive to gas giant extrasolar planets. The 110 or so that we have discovered probably represent the relatively close-in subset of the population of gas giant planets. Here we consider some of the questions that arise from the study of the properties of this subset. In particular we focus on the semimajor axis of extrasolar planets. Values of semimajor axis are a key parameter because observationally they are well determined and theoretically they are a key test of planet formation and migration. While many features of the semimajor axis distributions are well produced by models, features such as the apparent drop in eccentrities and metallicities towards large values of semimajor axis are not yet reproduced by simulations. A full understanding of the statistics of extrasolar planets awaits the discovery and study of a much broader range and larger sample of planets as well as continued intensive work on numerical simulations to produce more physical models of formation, migration and interaction.
\end{abstract}

\section{INTRODUCTION}

Ten years ago it would have the stuff of crazy wild dreams to imagine being asked to give a talk about "What is unknown about the statistics of extrasolar planets". My allocated title implies that this field is already well developed. The discovery of the 100th extrasolar planet suggests that the sample is large enough to make reasonably broad inferences about the population of planets as a whole. While I will argue that this is not true it is fun to consider a moment longer what astonishing progress has been made. In 1994 Geoff Marcy gave a somewhat despairing talk on his and Paul Butler's searches for brown dwarfs[12]. While this was an excellent much needed conference that probably organised the community for the era of discovery, the bottom line of this conference titled "The bottom of the main sequence and beyond" was that there were no brown dwarfs and certainly no planets. We conference participants passionately believed in the exciting future to come, though I don't think any of us would have predicted the field blossoming so quickly. Back then the idea of more than 100 extrasolar planets, as well as the current plans for Darwin/TPF, would have seemed utterly preposterous.

The confirmation of extrasolar planets by radial velocity, transit and astrometry has provided the motivation and justification for the next generation of ground (CELT, OWL) and space-based missions (MOST, SIRTF, COROT, Kepler, JWST, GAIA, SIM, Darwin/TPF). Nonetheless there are very severe biases in the discoveries made so far. While in many fields a sample of 100 objects might suffice to satisfy our curiosity 
the key difference is that extrasolar planets represent the possibilty to investigate life elsewhere and the next frontier in human exploration. The human race desires and thrives on discovery and exploration. So far we have experience of one planet and have a strong desire (and perhaps necessity) to explore other planets. The scientific and cultural interest in this field mean that based on the bibliography of 'The Extrasolar Planets Encyclopaedia"'[18] the field is now producing more than 500 papers per year. However, none of the known extrasolar planets are equivalent to any of the planets in our Solar System, thus it seems apt to quote caution from Alexander Pope in his Essay on Criticism "A little knowledge is a dangerous thing: drink deep, or taste not the Pierian spring; there shallow draughts intoxicate the brain, and drinking largely sobers us again."

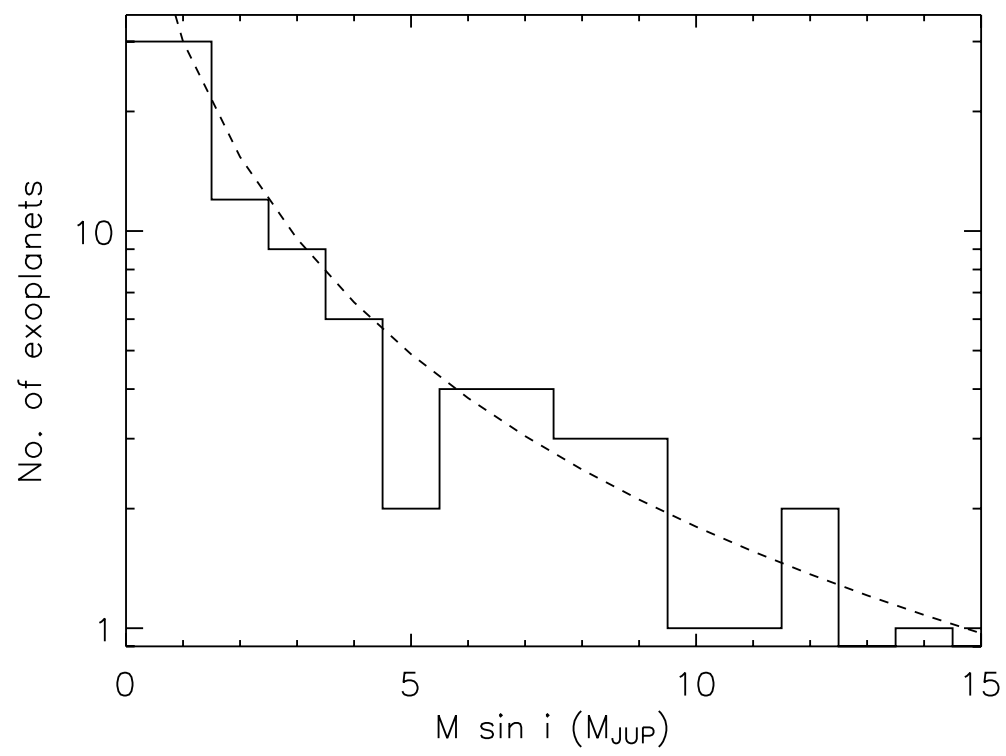

FIGURE 1. Number versus companion mass for all radial velocity extrasolar planets as given by [13]. The dashed line depicts the number of extrasolar planets proportional to Mass to the power -1.6 (not corrected for sensitivity function) and is normalised to fit through the mass bin of 1-2 M JUP sini i assumed to be reasonably complete for the sample of known extrasolar planets.

\section{WHAT IS AN EXTRASOLAR PLANET?}

With any new field nomenclature is important, in our case particularly crucial, since the information we have about extrasolar planets is all indirect. While we have confidence in the various indirect techniques that have been used we still await the detection of photons directly from an extrasolar planet. The word planet means 'wanderer' in Greek, thus planets move on the sky relative to the fixed background stars. This definition needs updating since with modern equipment even quite distant galaxies can be seen to move. Discussions about the status of Pluto and 'free-floating planets/brown dwarfs' [16] describe the issues. According to the Oxford English Dictionary (http://oed.com) the old astronomical meaning of planet 'A heavenly body distinguished from the fixed stars 
by having an apparent motion of its own among them; each planet, according to the Ptolemaic system, being carried round the earth by the rotation of the particular sphere or orb in which it was placed.' has evolved to 'The name given to each of the heavenly bodies that revolve in approximately circular orbits round the sun (primary planets), and to those that revolve round these (secondary planets or satellites)'. More specific definitions are provided elsewhere, e.g., http://dictionary.com: 'A non-luminous celestial body larger than an asteroid or comet, illuminated by light from a star, such as the sun, around which it revolves.' The IAU working group on extrasolar planets provides a half page working definition of extrasolar planet and a working list of candidate extrasolar planets (www.ciw.edu/boss/IAU/div3/wgesp).

Planetary mass objects may have already been imaged in young star forming regions (e.g. Tamura et al.[22]). Apart from the masses of these objects being very dependent on poorly constrained theoretical models, the 'free-floating' nature of these objects means they fall outside the currently accepted notion of extrasolar planet. An important strand in most definitions is the concept that to be a 'planet' an object must be in orbit around a 'star'. This proximity to a much brighter object as well as their relative faintness makes planets so difficult to find. Discovery would be simplified were it possible to directly image extrasolar planets. The best opportunity so far is perhaps the controversial planet around Epsilon Eridani. At only 3.2 pc, it should soon become feasible to image this object although with a separation of 1 arcsecond and a magnitude difference of 15 (a factor of 1,000,000 in brightness). While this observation is at the limit of current technology, a number of inovative techniques seem promising(e.g., [4]).

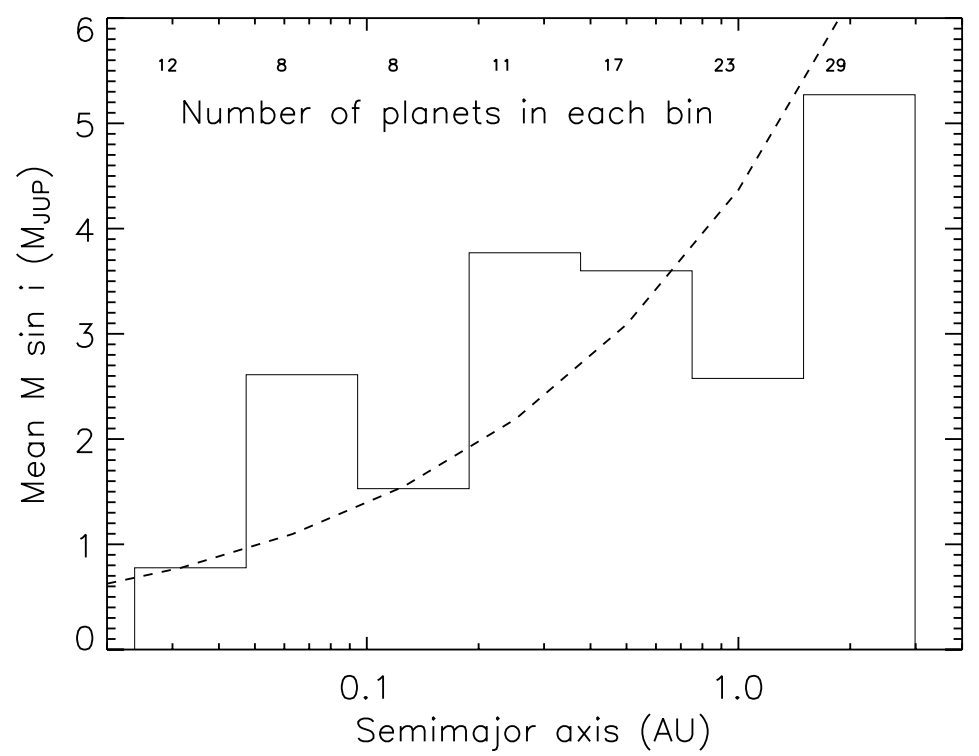

FIGURE 2. The mean mass (MJUP sin i) of extrasolar planets plotted with semimajor axis. The dotted line represents a crude approximation of the sensitivity function of the Doppler technique (semimajor axis to the power 0.5 ). 


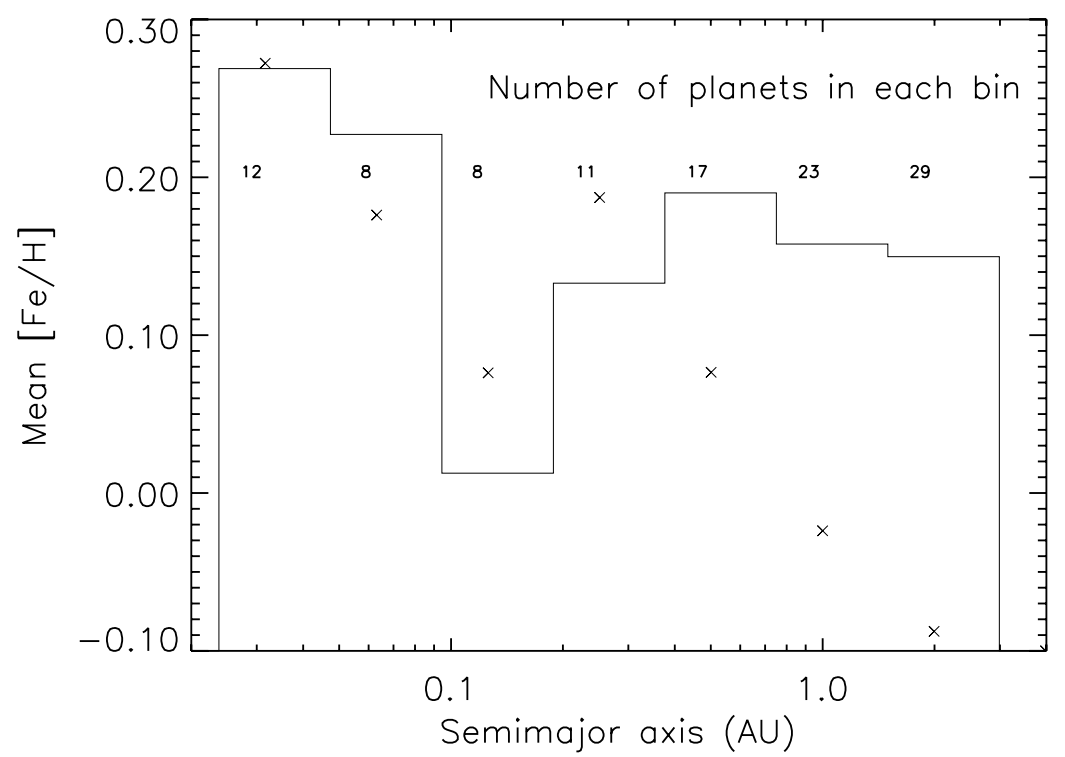

FIGURE 3. Mean spectroscopic metallicities of the primaries of extrasolar planets plotted as a function of semimajor axis. The crosses represent the low mass (0.1-1.1 MJUP $\sin$ i) third of detected extrasolar planets.

\section{BROWN-DWARF DESERT / PLANET JUNGLE AROUND SOLAR-TYPE STARS}

1995 was a watershed year, not just for extrasolar planets but also for brown dwarf research. A conference on cool stars in Florence saw the announcement of the extrasolar planet 51 Peg b as well as the brown dwarf Gl229B. Brown dwarfs bridge the gap between stars and planets. Too small and cool to be a star and sustain thermonuclear hydrogen burning but yet too massive to be a planet. Whilst a steady refinement of radial velocity searches means they are spectacularly sensitive to extrasolar planets, such searches are actually far more sensitive to the presence of brown dwarfs which have hardly been found. For many years it was expected that planets would be found by extending radial velocity searches of brown dwarfs to lower masses. However, while stellar radial velocity companions are relatively abundant, there is a relative deficit of companions from around 100 to $10 \mathrm{M}_{\mathrm{JUP}}$, approximately the brown dwarf regime. However, once sensitivity to below $10 \mathrm{M}_{\mathrm{JUP}}$ is achieved, Fig. 1 indicates how detections rapidly increase. This sharply rising detection rate at low masses is found against a sensitivity function for finding planets that falls in proportion to mass. 


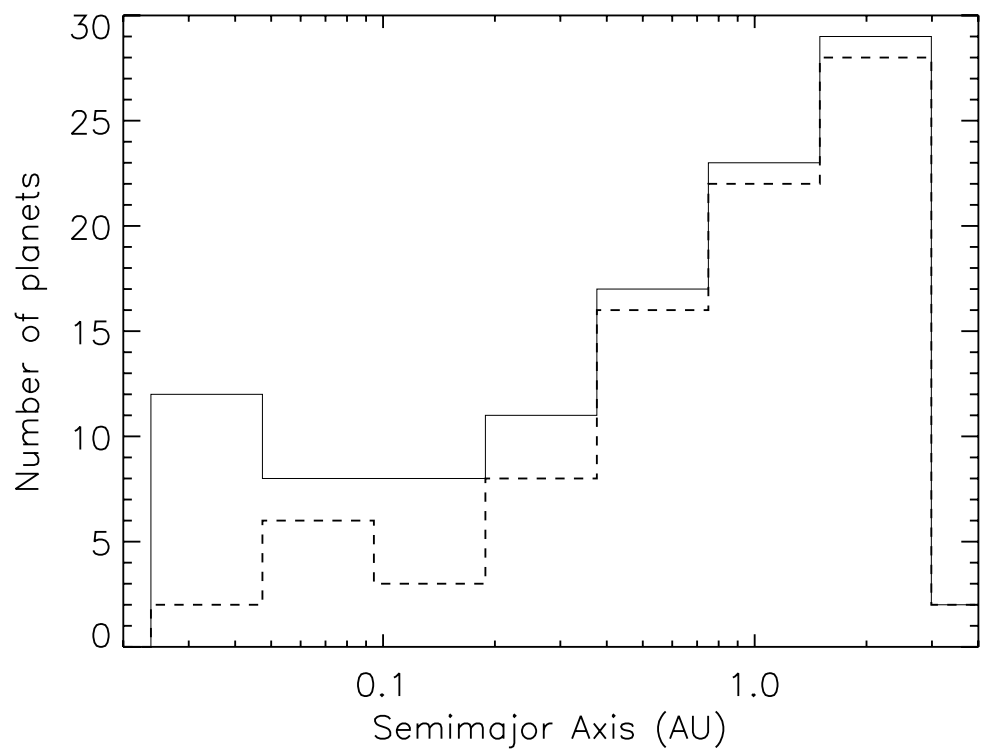

FIGURE 4. The number of extrasolar planets discovered with semimajor axis: solid line (all extrasolar planets), dashed line (those with masses above $0.8 \mathrm{MJUP}_{\mathrm{JU}} \sin \mathrm{i}$ ).

\section{WHY ARE EXTRASOLAR PLANETS SO DIFFERENT FROM SOLAR SYSTEM PLANETS?}

While the discovery of 51 Peg b was a landmark, as with the earlier discovery of planets around pulsars it was met with scepticism in part because of the difficulties of the measurement but as much because 51 Peg b seemed to have nothing in common with our Jupiter (apart from mass). Its orbit seemed to require radical new ideas about the formation of planets; though in fact all that was required was the rediscovery of the robust theoretical concept of inward planetary migration driven by tidal interactions with the protoplanetary disk[8]. Thus while such a large mass planet could not form in the glare of radiation from its Sun, it was entirely plausible that it had migrated into position through the disk of material around $51 \mathrm{Peg}[10]$. Although $51 \mathrm{Peg}$ b-like objects dominated the early discoveries, other types of planets are much more common. Of the 110 or so extrasolar planets that have been discovered the 51 Peg b-like planets (35 day orbital periods) represent a class of planets circling about $1 \%$ of stars[14]. The $51 \mathrm{Peg} b$ class were found first because the radial velocity technique is biased towards relatively heavy planets close to their stars, as they produce the largest signal. This makes these planets easiest to find. As more planets are discovered other types of biases in our understanding of extrasolar planets resulting from our experimental sensitivity will start to reveal themselves. Fig. 2 shows how the average mass of extrasolar planets increases with semimajor axis in accordance with the changing sensitivity of the radial velocity technique. This suggests that the long-term stability of the radial velocity searches is excellent providing the mass function of extrasolar planets is approximately constant 
with semimajor axis[1]. Fig. 2 is thus very encouraging for the potential detection of Solar System like extrasolar planets.

The extrasolar planets discussed and plotted in this paper all come from the compilation of Marcy et al. [13] and were all detection by the radial velocity technique. However, they are not discovered from a single well documented and quantified methodology. The compilation relies on a number of different ongoing surveys operating with different samples, sensitivities, instruments, scheduling, strategies and referencing techniques. Cumming et al.[2] has thoroughly investigated the observational biases inherent in the Lick and Keck surveys but has yet to report findings for the bulk of detected extrasolar planets. So far none of the surveys have the $3 \mathrm{~m} / \mathrm{s}$ precision over 15 years necessary to detect Jupiter and thus do not yet constrain the frequency of Solar Systems analogs. The relatively large number and fraction of planets that we have discovered before achieving sensitivity to our own Solar System together with the wide range of parameters discovered suggests that planetary formation and survival are robust. This seems to be borne out by theoretical work, which indicates that planet formation is an 'easy come, easy go' business, with many planets created and many destroyed, and with an important minority - including our own Jupiter - surviving[23]. Simulations, e.g. [1], suggest that gas giant planets will form at around 5 au. Over the next decade radial velocity searches should be well placed to constain such predictions. So far the compilation of extrasolar planets offers little constraint on extrasolar planets

around $\mathrm{O}, \mathrm{B}, \mathrm{A}$ and $\mathrm{M}$ stars,

beyond 4 au,

less massive than Saturn,

in regions outside the Solar Neighbourhood (e.g. clusters, bulge, halo),

in binary systems,

multiple systems.

\section{WHAT IS THE EXTRASOLAR PLANETARY MASS FUNCTION?}

Fig. 1 shows the mass function for extrasolar planets is rising fairly steeply towards lower masses. The simulations of Tabachnik \& Tremaine[21] and Zucker \& Mazeh[24] using around 60 planets favour a flatish mass distribution. The Bayesian approach[3] promises to incorporate a detailed knowledge of detection sensitivities. So far, the relatively small numbers of objects as well as the selection biases preclude much confidence in a particular value of the mass function. Nontheless, it is clear that we find an increasing number of objects towards lower masses which is consistent with our expectations based on our Solar System as well as simulations of planet formation. 


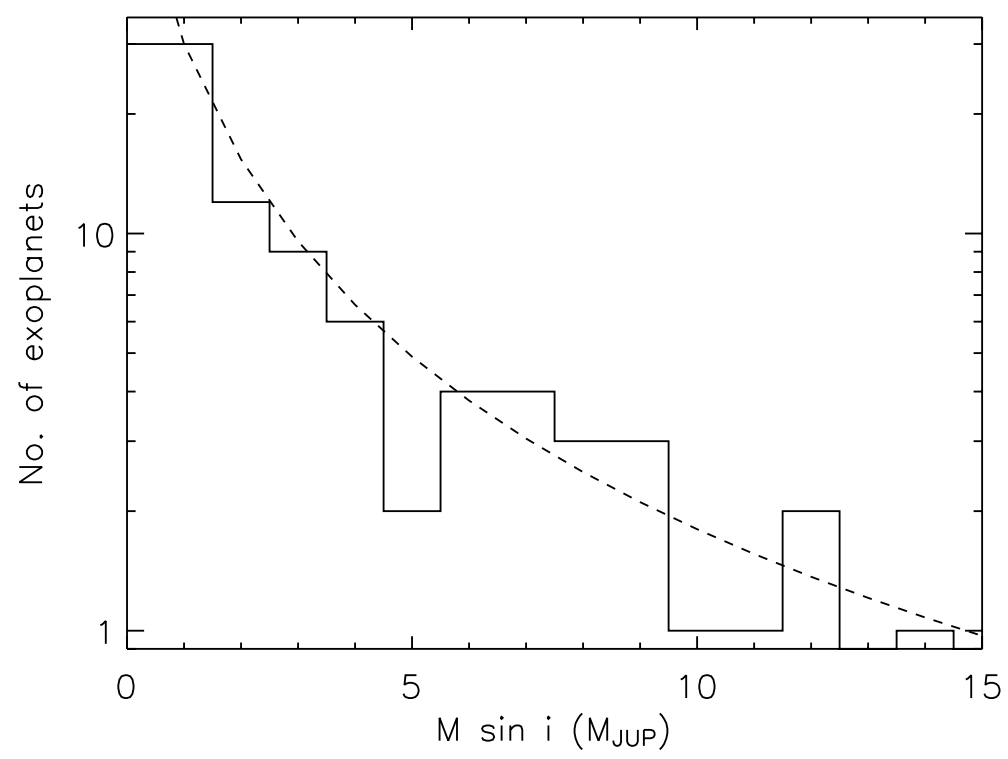

FIGURE 5. Mean spectroscopic metallicities of the primaries of extrasolar planets plotted as a function of extrasolar planet masses MJUP $\sin$ i.

\section{EVIDENCE FOR MIGRATION - SEMIMAJOR AXIS AND METALLICITY DISTRIBUTIONS?}

The distribution of extrasolar planets with semimajor axis in Fig. 4 suggests that extrasolar planets show key differences with semimajor axis. The solid line may suggest two separate features in the extrasolar planet semimajor axis distribution. A peak of shortperiod extrasolar planets is seen in the 51 Peg-type objects, then a dearth, followed by an smooth rise in the number of extrasolar planets toward longer periods[9]. However, except for a peak at 3 days [14], the short-period peak does not appear to be so significant when a completeness correction is made. The dotted line represents all extrasolar planets with masses greater than $0.8 \mathrm{M}_{\mathrm{JUP}} \sin \mathrm{i}$ and suggests that the short-period peak may well be a selection effect. The rise in the number of extrasolar planets towards larger semimajor axes is becoming more apparent as more are discovered and is well reproduced by extrasolar planet migration scenarios which envisage planets migrating inwards[1] as well as outwards[15].

An important characteristic of a star is its metallicity. Gonzalez[5] found extrasolar planet host stars to be metal-rich. This conclusion has been confirmed by many authors with different samples, methodologies and spectral synthesis codes (e.g., [7], [19]). Fig. 3 shows just how metal-rich the extrasolar planet primaries are. Only a single bin is around solar metallicity. All other bins are at least 0.1 dex above the solar; whereas the Sun and other solar type dwarf stars in the solar neighbourhood have an average metallicity of 0 or even slightly less[17]. The probability of detecting an extrasolar planet is proportional to its metallicity. By a metallicity of +0.3 dex the frequency of stars with 


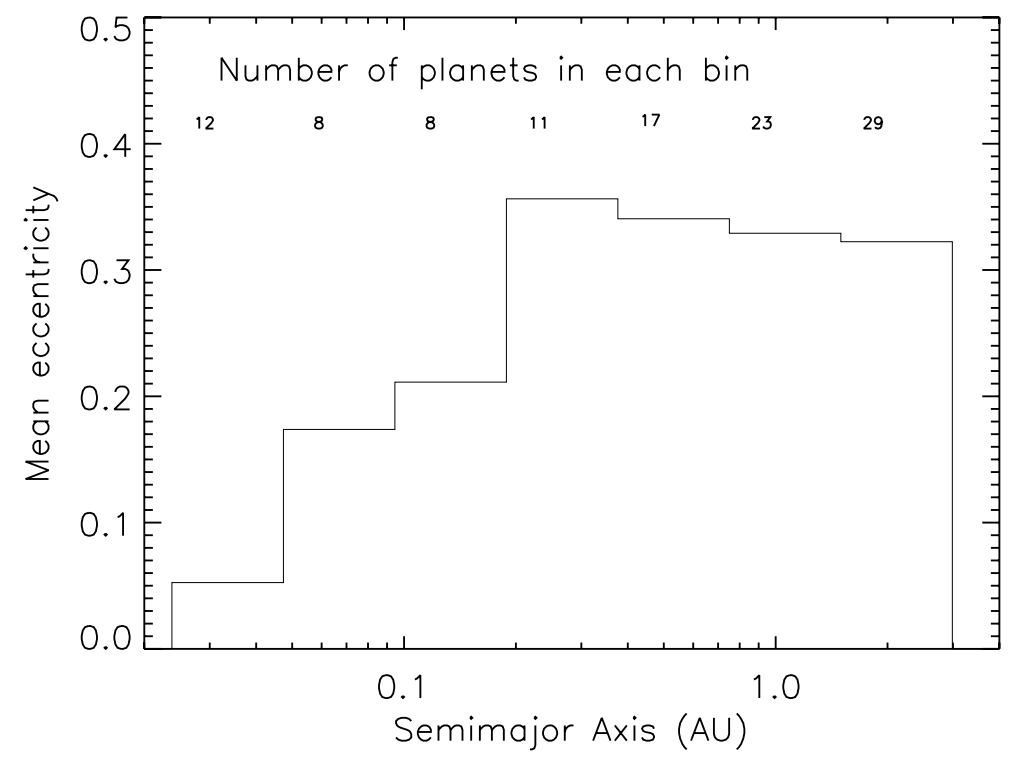

FIGURE 6. Eccentricity versus semimajor axis for extrasolar planets.

extrasolar planets is effectively $100 \%$. This result, representing the only link between the presence of planets and a stellar photospheric feature is explained by the classical view that giant planets are formed by runaway accretion of gas on to a 'planetesimal' having up to 10 Earth masses. In such a case, we can expect that the higher the proportion of dust to gas in the primordial cloud (i.e. metals), and consequently in the resulting protoplanetary disc, the more rapidly and easily may planetesimals, and subsequently the observed giant planets be built.

So far, it seems that the higher metallicity of most planet-harbouring stars arises because high metallicity environments have a higher probability of planet formation and migration. The relatively low metal content of the Solar System may be consistent with the relative lack of migration[11]. Thus it appears that analagous to the early detections of 51 Peg b-type extrasolar planets we are finding a surfeit of extrasolar planets around metal-rich stars because they are easier to detect. The idea that migration is dependent on metallicity also seems to be borne out by the overall decrease in metallicity for increasing semimajor axis. Given that the best explanation for the close-in planets is migration and that migration is modelled to be mass dependent, it is interesting to see if there is any mass dependency. The crosses in Fig. 3, represent the low-mass extrasolar planets (less than $1.1 \mathrm{M}_{\mathrm{JUP}}$ sin i) and suggest the slight decline to long periods is contributed primarily by the low-mass third of the extrasolar planet sample. This result is as expected by migration theories which predict more migration of lower mass objects. One might expect to see a relationship between metallicity and mass though yet none is readily apparent, e.g. Fig. 5. Such results are very preliminary and need confirmation with more extrasolar planets and higher precision metallicities. 


\section{WHY DON'T EXTRASOLAR PLANETS HAVE CIRCULAR ORBITS LIKE OUR SOLAR SYSTEM?}

Apart from the short-period extrasolar planets whose orbits are circularised by the tidal pull from their parent star, Fig. 6 shows the eccentricity of extrasolar planet orbits is much higher than in the Solar System. Eccentricities rise steeply out to semimajor axis values of around $0.2 \mathrm{au}$ at which point a mean eccentricity of around 0.35 is reached. This mean eccentricity shows a slight decline out to several au. Fig. 7 suggests that metallicity does not play an important role in the determination of eccentricity. Fischer et al.[6] find that it is rather close to that observed for stars. According to our paradigm of planetary formation a planet (formed in a disk) should keep relatively circular (low eccentricity) orbit. In order to boost extrasolar planet eccentricities it is necessary to imagine interactions between multiple planets in a disk and between a planet and a disk of planetesimals and perhaps the influence of a distant stellar companion. In fact dynamical interactions between planets seem inevitable since even with the fairly poor sampling of known extrasolar planets, 10 multiple systems have already been found. Of these a number are in resonant orbits. Thus 'dynamical fullness' is probably important and suggests that interactions play a vital role in determining the properties of many extrasolar planets. These orbital complexities mean that to understand extrasolar planets more generally, it will be necessary to find all the main components in planetary systems. This will require using results from different techniques, particularly radial velocity and astrometric, to disentangle the various planetary components in orbit around nearby stars. Fischer et al. (2003) suggest that selection effect may play a role in the high eccentricities observed among the single extrasolar planets discovered to date. Most known extrasolar planets reside within 3 au due to the limited duration (10 yr) of the Doppler surveys. Thus the planets detected to date represent a subset that ended up within 3 au. Giant planets within 3 au may systematically represent the survivors of scattering events in which the other planet was ejected while extracting energy from the surviving planet and throwing it inward. This would give rise to us systematically detecting the more massive, surviving planet residing in an orbit with a period less than 10 years.

\section{CONCLUSIONS}

Detecting the true distributions of extrasolar planets for the radial velocity technique will require a quantitative knowledge of detectability function for the radial velocity technique. Detectability can easily be corrected for the enhanced sensitivity of the radial velocity technique to large mass planets with short orbital periods. However, corrections for sampling, duration of observations, velocity jitter and differing sensitivity of different surveys are much more subtle. The move to the automation of radial velocities should help in the quantification and optimisation of radial velocity surveys. Overall our sample of extrasolar planets is still subject to biases which have not yet been quantified and not representative of planetary systems in general which we expect to include terrestrial

planets and ocean planets as well as gas giants. Nonetheless, if knowledge in this field 


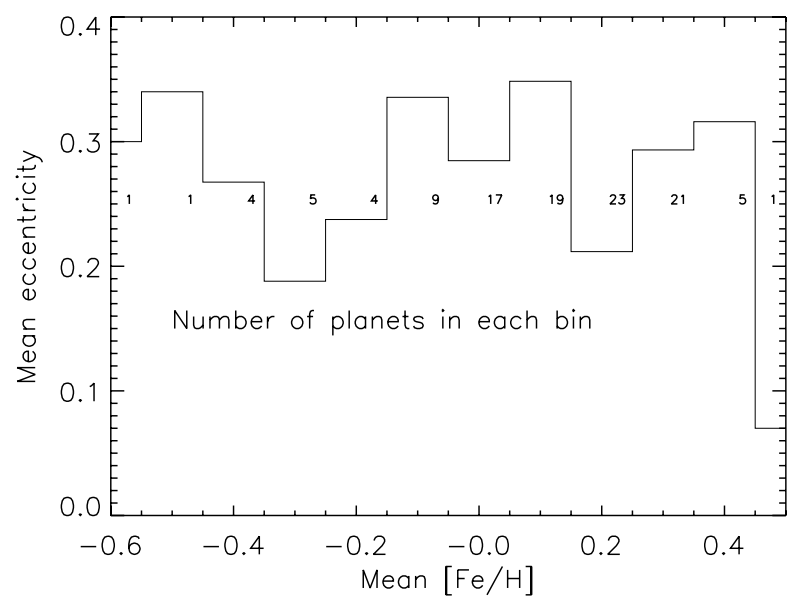

FIGURE 7. Mean eccentricities as a function of spectroscopic metallicities of the primaries of extrasolar planets.

can progress in the next decade as fast as the last, an incredible era of discovery awaits.

\section{REFERENCES}

1. Armitage P.J., Livio M., Lubow S.H., Pringle J.E., 2002 MNRAS, 334, 248

2. Cumming A., Marcy G.W., Butler R.P., 1999, ApJ, 526, 896

3. Cumming A., Marcy G.W., Butler R.P., Vogt S.S., 2003, ASP Conf. Ser. 294, p27, eds. Deming D., Seager $\mathrm{S}$.

4. Danchi W.C., Deming D., Kuchner M.J., Seager S., 2003, ApJ, 597, 57L

5. Gonzalez G., 1997, MNRAS, 285, 403

6. Fischer D.A. et al., 2003, ApJ, 586, 1394

7. Fischer D.A., Valenti J.A., Marcy G.W., 2004, IAU 219, in press, ed. Dupree A.

8. Goldreich P., Tremaine S., 1980, ApJ, 241, 425

9. Jones et al., 2003, MNRAS, 341, 948

10. Lin D.N.C., Bodenhimer P., Richardson D.C., 1996, Nature, 380, 606

11. Lineweaver C.H., 2003, Icarus, 151, 307

12. Marcy G.W., Butler R.P., 1995, The Bottom of the Main Sequence and Beyond, p98, ed. Tinney, C., Springer-Verlag,

13. Marcy G.W., Butler R.P., Vogt S.S., Fischer D.A., et al., 2003, http://extrasolar planets.org

14. Marcy G.W., Butler R.P., Vogt S.S., Fischer D.A., 2004, ASP Conf Ser., in press, eds Beaulieu J-P., Lecavelier des Etangs A., Terquem C.

15. Masset F.S., Papaloizou J.C.B., 2003, ApJ, 588, 494

16. McCaughrean M.J. et al., 2001, Science 291, 1487

17. Reid I.N., 2002, PASP, 114, 306

18. Schneider J., 2003, http://www.obspm.fr/encycl/encycl.html

19. Santos N. et al. 2003, A\&A, 398, 363

20. Santos N. et al. 2004, A\&A, in press

21. Tabachnik S., Tremaine S., 2002, MNRAS, 335, 151

22. Tamura M., Itoh Y., Oasa Y., Nakajima T., 1998, Science, 282, 1095

23. Trilling D., Lunine J.I., Benz W., 2003, A\&A, 394, 241

24. Zucker S., Mazeh T., 2002, ApJ, 568, 113L 